\title{
Matrix Algebra and Eigenvalues for the Bead/Spring Model of Polymer Solutions
}

\author{
Jeffrey T. Fong* \\ Institute for Basic Standards, National Bureau of Standards, Washington, D.C. 20234 \\ and
}

Anton Peterlin

Institute for Materials Research, National Bureau of Standards, Washington, D.C. 20234

(February 12, 1976)

\begin{abstract}
Based on purely matrix-algebraic arguments, we prove three new results on the eigenvalue problem [Zimm, 1956] arising from modeling the bulk hydrodynamic and dielectric properties of very dilute polymer solutions:

1. Let $N$ be the number of identical segments joining $N+1$ beads of a bead/spring model for any polymer molecule. Then for any $N$, the well-known hydrodynamic interaction matrix $\stackrel{H}{=}$ is positive definite if the so-called interaction parameter $h^{*}$ is less than $(2 \sqrt{2}+1) / 7$ or 0.547 .

2. Let $\stackrel{A}{=}$ be the tridiagonal matrix governing the elastic link force on each bead. Then if $\stackrel{H}{=}$ is positive definite, there exists a matrix $\underline{\underline{Q}}$ such that $\underline{\underline{Q}}^{-1} \underline{\underline{H}} \underline{\underline{A}} \underline{\underline{Q}}=\underline{\underline{Q}}^{\mathrm{T}} \underline{\underline{\mathrm{A}}} \underline{\underline{Q}}=\underline{\underline{\Lambda}}$, where $\underline{\underline{\Lambda}}$ is a diagonal matrix.

3. (Equivalence Statement due to Lodge and $\mathrm{Wu}$ [1971]). The nonzero eigenvalues of the matrix product $\stackrel{\mathrm{H}}{=} \stackrel{\mathrm{A}}{=}$ are completely determined by solving the eigenvalue problem associated with a symmetric matrix $\underline{\underline{\mathrm{S}}}$, where $S_{i j}=H_{i j}+H_{i-1, j-1}-H_{i-1, j}-H_{i, j-1}, i, j=1,2, \ldots, N$.
\end{abstract}

To illustrate the significance of these results, numerical output for $N$ as large as 300 based on a Fortran program for several values of $h^{*}$ is given.

Key words: Bead/spring model; dilute polymer solutions; eigenvalue; hydrodynamic interaction; matrix algebra; necklace model; polymer physics.

\section{Statement of Problem}

Some twenty years ago, Zimm [1] $]^{1}$ formulated a linear, second-order partial differential equation for a distribution function $\psi$ which depends on time and $3(N+1)$ coordinates $x_{0}, y_{0}, z_{0} \ldots, x_{N}, y_{N}$, $z_{N}$, of the $N+1$ beads, for modeling the bulk behavior of very dilute polymer solutions under the influence of external force, Brownian motion, and hydrodynamic interaction among the beads of the necklace model. The mechanical model for each polymer molecule is that of a chain of $N$ identical, ideally elastic segments joining $N+1$ identical beads with complete flexibility at each bead. Two length parameters are of interest in this model: $a_{h}$, the so-called hydrodynamic radius of the bead, and $b_{0}$, the root mean square of the segment length. The ratio $a$ of the two length parameters $(a=$ $a_{h}\left(b_{0}\right.$ ), and the number $N$ of elastic segments completely characterize the mathematical problem

\footnotetext{
*On leave during 1975-76 as a ComSci Fellow, Nuclear Regulatory Comm., Wash., D.C. 20555.

1 Figure in brackets indicate the literature references on page 281.
} 
through the following two symmetric, square matrices of order $N+1$ :

$$
\stackrel{\mathrm{A}}{=} \quad=\left[\begin{array}{rrrrrr}
1 & -1 & 0 \ldots & 0 & 0 & 0 \\
-1 & 2 & -1 \ldots & 0 & 0 & 0 \\
0 & -1 & 2 \ldots & 0 & 0 & 0 \\
\ldots \ldots \ldots & \ldots \ldots \ldots \ldots \ldots \\
0 & 0 & 0 \ldots & 2 & -1 & 0 \\
0 & 0 & 0 \ldots & -1 & 2 & -1 \\
0 & 0 & 0 \ldots & 0 & -1 & 1
\end{array}\right]
$$

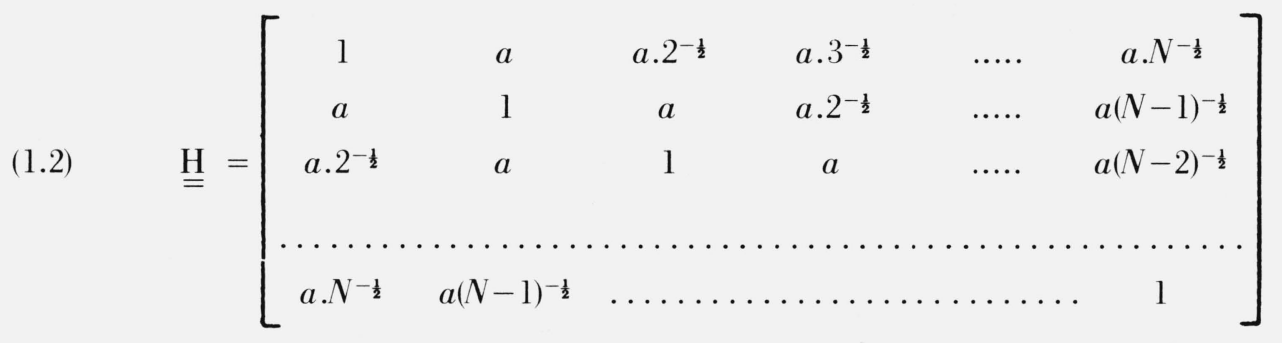

The symmetry of the two matrices, $\underset{=}{A}$ and $\underline{\underline{H}}$, and an assumption based on physical arguments that the eigenvalues of the matrix product $\stackrel{\mathrm{H}}{=} \stackrel{\mathrm{A}}{=}$ are distinct, led to an eigenvalue problem with a coordinate transformation matrix $\underline{\underline{Q}}$ such that a similarity transformation with $\underline{\underline{Q}}$ diagonalizes $\underline{\underline{H}} \stackrel{\underline{A}}{\underline{\underline{A}}}$ and congruent transformations with $\underline{\underline{Q}}$ diagonalize $\underset{\underline{\mathrm{A}}}{\underline{\text { and }}} \underline{\underline{\mathrm{H}}}$ as follows:

$$
\begin{aligned}
& \underline{\underline{Q}}^{-1} \stackrel{\mathrm{H}}{=} \stackrel{\underline{\mathrm{Q}}}{=} \underline{\underline{\Lambda}}=\underline{\underline{\Lambda}}, \\
& \underline{\underline{Q}}^{\mathrm{T}} \quad \stackrel{\mathrm{A}}{=} \stackrel{\underline{\mathrm{Q}}}{=}=\stackrel{\mathrm{M}}{=} \text {, } \\
& \underline{\underline{Q}}^{-1} \underline{\mathrm{H}}\left[\underline{\underline{Q}}^{-1}\right]^{T}=\underline{\underline{\mathrm{N}}}=\underline{\underline{\Lambda}} \underline{\underline{\mathrm{M}}}^{-1},
\end{aligned}
$$

where $\underline{\underline{\Lambda}}=\operatorname{diag}\left(\lambda_{0}, \lambda_{1}, \ldots, \lambda_{N}\right), \underline{\underline{M}}=\operatorname{diag}\left(\mu_{0}, \mu_{1}, \ldots, \mu_{N}\right)$, and $\underline{\underline{N}}=\operatorname{diag}\left(\nu_{0}, \nu_{1}, \ldots, \nu_{N}\right)$, are diagonal matrices, and the superscripts ${ }^{-1}$ and $t$ in eqs (1.3) through (1.5) denote the inverse and the transpose of a matrix, respectively. A closed form solution for a specific linear laminar flow with a transverse gradient constant over all beads, was first given by Peterlin and appeared at the end of Zimm's paper [1, p. 278] as a note added in proof. Computationally speaking, the modeling problem is reduced to the construction of the transformation matrix $\underline{\underline{Q}}$ such that both $\underline{\underline{\Lambda}}$ and $\underline{\underline{M}}$ are known for constructing the distribution function $\psi$.

The purpose of this paper is to derive some new results based on matrix algebraic arguments and another obvious property of the matrix $\stackrel{\mathrm{H}}{=}$, namely, its positive-definiteness under certain conditions, which Zimm [1] failed to exploit. In section 2, we first show that $\underline{\underline{H}}$ is positive definite if the parameter a is positive and is bounded from above by a constant $a_{\max }$ which equals 1.0 for $N=$ 1, 0.872 for $N=2,0.815$ for $N=3$, and 0.773 for $N \geq 4$. In section 3 , we prove our main result 
that for positive definite $\underline{\underline{H}}$, there exists some $\underline{\underline{Q}}$ such that $\underline{\underline{\Lambda}}=\underline{\underline{M}}$ and $\underline{\underline{N}}=\underline{\underline{1}}$, i.e., $\lambda_{j}=\mu_{\mathrm{j}}$ and $\nu_{j}$ $=1$ for $j=0,1,2, \ldots, N$. In section 4 , we furnish an algebraic proof as an alternate to the proof of a result due to Lodge and $\mathrm{Wu}[2]$, which we subsequently use in calculating the eigenvalues of the matrix product $\underline{\underline{\mathrm{H}}} \underset{\mathrm{A}}{=}$. In section 5 , we discuss the significance of our three mathematical results.

To illustrate the significance, we include in an appendix a sample Fortran program and output for several values of the nondimensional parameter $a$ and for $N$ as large as 300 .

\section{Positive Definiteness of the Interaction Matrix}

As shown in (1.2), the so-called interaction matrix $\underline{\underline{H}}$ is not only symmetric, but also a "stripe matrix," i.e., all entries along a line parallel to the main diagonal are equal to one another. Furthermore, $\underline{\underline{\mathrm{H}}}$ depends on a scalar parameter $a$, which is required to be positive $(\mathrm{a}>0)$ from physical considerations. ${ }^{2}$ We now wish to show that, for any given order of the matrix $\underline{\underline{H}}$, there is an upper bound $a_{\max }$ for the parameter $a$ such that $\underline{\underline{H}}$ is positive definite for $0<a<a_{\max }$.

Since $\underline{\underline{H}}$ is a stripe matrix, an application of a standard result in matrix algebra ${ }^{3}$ implies the corollary that $\underline{\underline{H}}$ is positive definite, if and only if, the determinant ${ }_{\underline{H}}^{\underline{H}}$, i.e., det $\underline{\underline{H}}$, is positive for a specific order $N+1$ as well as for all orders less than $N+1$. It is convenient to introduce a new matrix $\underline{\underline{B}}$ and a new positive constant $c$ as defined below:

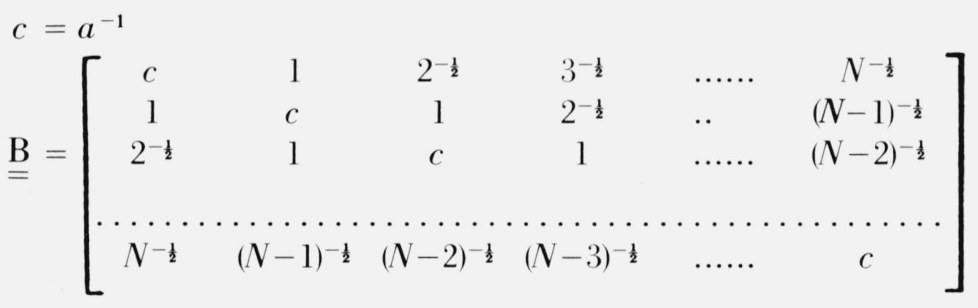

Since det $\underline{\underline{B}}=c^{N+1}$ det $\underline{\underline{H}}, c>0$, our problem is reduced to the study of the positivity of det $\underline{\underline{B}}$.

From the property of a determinant that its value is unaltered if any multiple of one line is added to a different, parallel line, ${ }^{4}$ let us perform the following operations on the matrix $\underline{\underline{B}}$ :

Step I Keep the first row of $\underline{\underline{B}}$ fixed, and replace the $j$ th row with the difference between the $j$ th and the $(j-1)$ th rows for $j=2,3, \ldots, N+1$.

Step 2 Keep the first column of the new matrix following step 1 fixed, and replace the $j$ th column of the new matrix with the difference between the $j$ th and the $(j-1)$ th columns of the new matrix for $j=2,3, \ldots, N+1$.

${ }^{2}$ We have already seen that by definition, $a\left(=a_{h} / b_{0}\right)$ is required to be positive if the hydrodynamic radius $a_{h}$ is positive. In Zimm's formulation [1, pp. $270-$ 271], the parameter is defined by the expression $\left(6 \pi^{3}\right)^{-1} \rho \eta^{-1} b^{-1}$, which is positive for $\rho>0, \eta>0$, and $b>0$.

see, e.g., Hohn [3, pp. 2̧r-200].

${ }^{4}$ See, e.g., Hohn [3, p. 37]. 
The resulting matrix, to be denoted by $\underline{\underline{B}}^{\prime}$, is again symmetric as shown below:

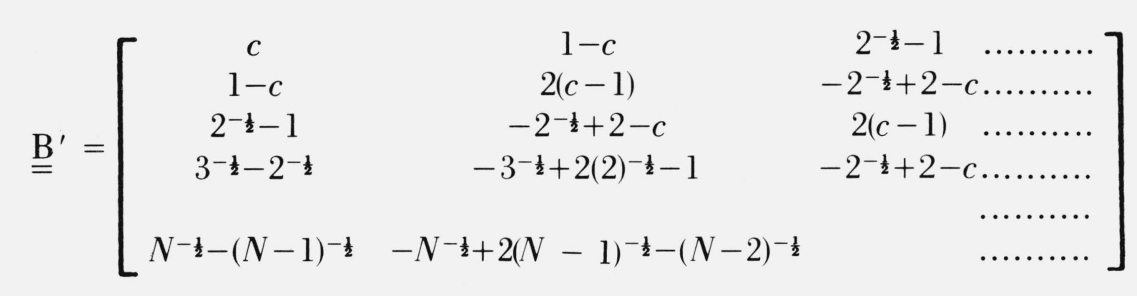

Since $\operatorname{det} \underline{\underline{B}}^{\prime}=\operatorname{det} \underline{\underline{B}}$, we have further reduced the study of the positive definiteness of the interaction matrix $\stackrel{\mathrm{H}}{=}$ to a study of the matrix $\mathrm{B}_{=}^{\prime} . \quad A s$ it will become clear very shortly, our derivation so far is intended to pave the way for the application of a sufficient condition for the positive definiteness of $\underline{\underline{H}}$ or $\underline{\underline{B}}^{\prime}$.

We now observe that for the lowest order of $\underline{\underline{H}}, \underline{B}$, or $\underline{B}^{\prime}$, i.e., all the $2 \times 2$ matrices given by (1.2), (2.2), or (2.3), the positivity of $\operatorname{det} \stackrel{\mathrm{H}}{=}$ (and thus det $\stackrel{\mathrm{B}}{=}$ or $\left.\operatorname{det} \mathrm{B}^{\prime}\right)$ always yields the condition that either $a<1$ or $c>1$. It follows that the symmetric matrix $\underline{B}^{\prime}$ has the property that all its diagonal elements are positive. A well-known theorem in matrix algebra states that a symmetric matrix with positive diagonal elements is positive definite if the matrix is "strongly diagonal dominant," i.e., the absolute value of the diagonal element at every row exceeds the sum of the absolute values of all off-diagonal elements in that row: ${ }^{5}$

$$
\left|B_{j j}^{\prime}\right|>\sum_{\substack{k=0,1, \ldots \\(k=j)}}^{N}\left|B_{j k}^{\prime}\right|, \quad \text { for } j=0,1,2, \ldots, N
$$

We denote the generic of $\underline{\underline{B}}^{\prime}$ as $B_{j k}, j, k=0,1,2, \ldots, N$.

To apply (2.4), we consider two ranges of the values of $c$ for which one can write down easily the $N+1$ inequalities:

Case l $\left(c \geq 2-2^{\left.-\frac{1}{2}\right)}\right.$ :

$$
\begin{aligned}
& j=0: \quad c \quad>c-\mathrm{N}^{-\frac{1}{2}} . \\
& j=1: \quad 2(c-1)>2(c-1)-(N-1)^{-\frac{1}{2}}+N^{-\frac{1}{2}} . \\
& j=2: \quad 2(c-1)>2(c-1)-(N-2)^{-\frac{1}{2}}+(N-1)^{-\frac{1}{2} .}
\end{aligned}
$$

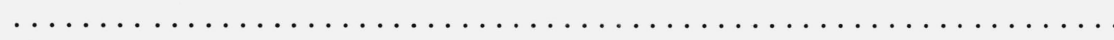

$$
\begin{aligned}
& j=N: \quad 2(c-1)>c-1 .
\end{aligned}
$$

Case $2\left(1<c<2-2^{-\frac{1}{2}}\right)$ :

$$
j=0: \quad c>c-N^{-\frac{1}{2}}
$$

\footnotetext{
${ }^{5}$ See, e.g., Schwarz [4, pp. 17-19].
} 


$$
j=1: \quad 2(c-1)>1-2^{-\frac{1}{2}}
$$

$(N=2)$,

$$
\text { or, } \quad 2(c-1)>2-3(2)^{-\frac{1}{2}}+3^{-\frac{1}{2}}
$$

$(N=3)$,

$$
\text { or, } \quad 2(c-1)>2-2(2)^{-\frac{1}{2}}-(N-1)^{-\frac{1}{2}}+N^{\frac{1}{2}}
$$

$(N \geqq 4)$.

$$
j=2: \quad 2(c-1)>3-2(2)^{-\frac{1}{2}}-c
$$$$
(N=2),
$$

$$
\begin{aligned}
& \text { or, } \quad 2(c-1)>5-3(2)^{-\frac{1}{2}}-2 c \\
& (N=3), \\
& \text { or, } \quad 2(c-1)>6-5(2)^{-\frac{1}{2}}-2 c+3^{-\frac{1}{2}} \\
& (N=4), \\
& \text { or, } \quad 2(c-1)>6-4(2)^{-\frac{1}{2}}-2 c-(N-2)^{-\frac{1}{2}}+(\mathrm{N}-1)^{-\frac{1}{2}} \\
& (N \geqq 5) . \\
& j=3: \quad 2(c-1)>3-2(2)^{-\frac{1}{2}}-c \\
& (N=3), \\
& (N=4), \\
& \text { or, } \quad 2(c-1)>5-3(2)^{-\frac{1}{2}}-2 c \\
& (N=5, \\
& \text { or, } \quad 2(c-1)>6-5(2)^{-\frac{1}{2}}-2 c+3^{-\frac{1}{2}} \\
& \text { or, } \quad 2(c-1)>6-4(2)^{-\frac{1}{2}}-2 c-(N-3)^{-\frac{1}{2}}+(N-2)^{-\frac{1}{2}} \\
& (N \geqq 6) \text {. } \\
& j=N: \quad 2(c-1)>3-2(2)^{-\frac{1}{2}}-\mathrm{c}
\end{aligned}
$$

\begin{tabular}{|c|c|c|c|}
\hline \multirow{2}{*}{ Number of Chains } & $a_{\max }$ & & $h_{\max }^{*}=2^{-1 / 2}\left(a_{\max }\right)$ \\
\hline & (Exact) & (Approx.) & (Approx.) \\
\hline$N=1$ & 1 & 1.0 & 0.707 \\
\hline$N=2$ & $\frac{1}{17}(12+2 \sqrt{2})$ & 0.872 & 0.617 \\
\hline$N=3$ & $\frac{3216+1278 \sqrt{2}-484 \sqrt{3}-288 \sqrt{6}}{4273}$ & 0.815 & 0.576 \\
\hline$N=4$ and above & $\frac{1}{7}(4+\sqrt{2})$ & 0.773 & 0.547 \\
\hline
\end{tabular}

What distinguishes the above two cases is the fact that for case 1 , every off-diagonal element of $\underline{\underline{B}}^{\prime}$ is negative, whereas for case 2 , care must be exercised on elements $B_{23}^{\prime}, B_{33}^{\prime} B_{34}^{\prime} \ldots \ldots B_{N, N-1}^{\prime}$, each of which equals $-2^{-\frac{1}{2}}+2-c$. An examination of inequalities (2.5) through (2.8) for case 1 implies that they are always satisfied for every $N$. On the other hand, for case 2, the controlling inequalities are given by (2.9), (2.10), and (2.11). Combining all results derived in this section, including (2.1) where $\mathrm{a}=c^{-1}$, we obtain various upper bounds $a_{\max }$ for the parameter $a$ for various $N$ as listed in table 1.

TABLE 1. Upper bounds for a or $\mathrm{h}^{*}$ for positive definite $\underline{\underline{\mathrm{H}}}$ 
We have included in table 1 a list of upper bounds for a parameter $h^{*}$ which appears frequently in the literature with $h^{*}=2^{-\frac{1}{2}} a$. Since most of the applications of the bead/spring model require more than four chains $(N \geq 4)$, the key result, $0<h^{*}<0.547$, a criterion which assures positive definiteness of $\underline{\underline{H}}$, will be used in the next section to achieve a significant simplification of Zimm's eigenvalue problem.

\section{Diagonalization-A Simplification of Zimm's Result}

The derivation of an upper bound for the parameter $a(0<a<0.773)$ or, equivalently, the parameter $h^{*}\left(0<h^{*}<0.547\right)$, with $h^{*}=2^{-\frac{1}{2}} a$, as shown in the last section, enables us to inject the positive definiteness of the matrix $\underline{\underline{H}}$ as an additional matrix property into Zimm's eigenvalue problem. We begin with another standard result in matrix algebra that any symmetric, positive definite matrix can be represented by the square of another symmetric matrix. ${ }^{6}$ Hence, for $0<h^{*}$ $<0.547$, the symmetric matrix $\underline{\underline{H}}$ is positive definite and there exists a symmetric $\underline{\underline{C}}$ such that $\underline{\underline{H}}=$ $\underline{\underline{C}}^{2}, \underline{\underline{C}}^{T}=\underline{\underline{C}}$. Since the matrix $\underset{\underline{A}}{=}$ as defined in (1.1) is symmetric, the matrix product $\underline{\underline{C}} \stackrel{\mathrm{A}}{=} \underline{\underline{C}}$ is also symmetric. By the well-known diagonalization theorem on symmetric matrices, ${ }^{7}$ we assert that there exist an orthogonal $\underline{\underline{U}}$ and a diagonal $\underset{\underline{D}}{=}$ such that

$$
\underline{\underline{\mathrm{C}}} \underline{\underline{\mathrm{A}}} \underline{\underline{\mathrm{C}}}=\underline{\underline{\mathrm{U}}}^{-1} \underline{\underline{\mathrm{D}}} \underline{\underline{\mathrm{U}}} ; \quad \underline{\underline{\mathrm{U}}}^{T} \underline{\underline{\mathrm{U}}}=\underline{\underline{\underline{1}}} .
$$

By choosing $\underline{\underline{Q}}$ as the matrix product $\underline{\underline{C}} \underline{\underline{U}}{ }^{-1}$ eqs (1.3) through (1.5) can be simplified as follows:

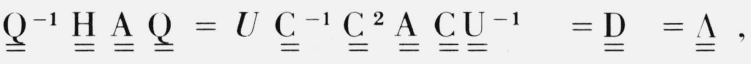

$$
\begin{aligned}
& \underline{\underline{Q}}^{T} \underline{\underline{\mathrm{A}}} \underline{\underline{\mathrm{Q}}}=\underline{\underline{\mathrm{U}}} \underline{\underline{\mathrm{C}}} \stackrel{\underline{\mathrm{A}}}{\underline{\mathrm{C}}} \underline{\underline{\mathrm{U}}}^{-1}=\underline{\underline{\mathrm{D}}}=\underline{\underline{\mathrm{M}}} \quad, \\
& \underline{\underline{Q}}^{-1} \underline{\underline{H}}\left(\underline{\underline{Q}}^{-1}\right)^{T}=\underline{\underline{\mathrm{U}}} \underline{\underline{C}}^{-1} \underline{\mathrm{C}}^{2} \underline{\underline{C}}^{-1} \underline{\underline{U}}^{-1}=\underline{\underline{1}}=\underline{\underline{\mathrm{N}}} .
\end{aligned}
$$

Thus we have shown that for $0<h^{*}<0.547$, there exists a transformation matrix $\underline{Q}$ such that $\underline{\underline{\Lambda}}=\underline{\underline{M}}$, and $\underline{\underline{N}}=\underline{\underline{1}}$.

\section{Equivalence of Two Eigenvalue Problems-An Algebraic Proof}

For the bead and spring model of dilute polymer solutions, the result of last section shows that it is sufficient to find the $N+1$ eigenvalues of the matrix product $\stackrel{\underline{\underline{H}}}{\underline{\mathrm{A}}}$ if the criterion $0<a<0.773$ or $0<h^{*}<0.547$ is satisfied. Since $\underline{\underline{\mathrm{A}}}$ is singular and all eigenvalues are distinct, det $(\underline{\underline{\mathrm{H}}} \underline{\underline{\mathrm{A}}})$ vanishes and exactly one of those $N+1$ eigenvalues must be zero, i.e., $\lambda_{0}=0$.

To find the other $N$ eigenvalues, $\lambda_{1}, \ldots, \lambda_{N}$, Lodge and $\mathrm{Wu}$ [2] introduced an equivalence statement which asserts that the eigenvalue problem for the unsymmetric product $\underline{\underline{H}} \stackrel{\mathrm{A}}{=}$ of order $N+$ 1, aside from the addition of a zero eigenvalue, is equivalent to that of a symmetric matrix

\footnotetext{
${ }^{6}$ See, e.g.. Halmos [2, p. 139].
${ }^{7}$ See, e.g.. Halmos [2, p. 157, bottom lines].
} 
$\underline{\underline{\mathrm{S}}}$ of order $N$, where the elements $S_{i j}$ of $\underline{\underline{\mathrm{S}}}$ are combinations of elements of $\underline{\underline{\mathrm{H}}}$ as follows:

$$
S_{i j}=H_{i j}+H_{i-1, j-1}-H_{i-1, j}-H_{i, j-1}, \quad i, j=1,2, \ldots, N
$$

The equivalence statement is important because the matrix $\underline{\underline{S}}$ for the reduced problem is symmetric and, therefore, computationally more attractive than the matrix product $\underline{\underline{\mathrm{H}}} \stackrel{\mathrm{A}}{=}$. To prove that statement, Lodge and $\mathrm{Wu}[2]$ introduced a nonsingular matrix $\stackrel{\mathrm{A}}{\epsilon}_{\epsilon}$ with elements $\left(A_{\epsilon}\right)_{i j}=A_{i j}$ for each $i, j=0,1, \ldots, N$, except for $i=j=0$ where $\left(A_{\epsilon}\right)_{00}=A_{00}+\epsilon^{2}$. Using a theorem ${ }^{8}$ on the continuity of eigenvalues, a triangular decomposition of the matrix $\underline{A}_{\epsilon}$, and a property of the determinant of a matrix product, it was shown that as $\epsilon \rightarrow 0$, the nonzero eigenvalues of $\underline{\underline{H}} \stackrel{\underline{A}}{\epsilon}$ approach those of $\underline{\underline{\mathrm{H}}} \underset{\mathrm{A}}{=}$ and $\underline{\underline{\mathrm{S}}}$.

The purpose of this section is to show that Lodge and Wu's equivalence statement is true by a purely algebraic argument without resorting to the continuity theorem involving the parameter $\epsilon$. We first observe that there exists a triangular decomposition of $\underline{\underline{A}}$ such that $\underline{\underline{A}}=\underline{\underline{L}}^{T} \underline{\underline{L}}$ :

$$
\underline{\underline{L}}=\left[\begin{array}{rrrrrr}
0 & 0 & 0 & \ldots & & \\
-1 & 1 & 0 & & & \\
0 & -1 & 1 & & & \\
& & & & 1 & 0 \\
0 & & & & -1 & 1
\end{array}\right]
$$

Let $\lambda_{i}$ be any nonzero eigenvalue of $\underline{\underline{\mathrm{H}}} \stackrel{\mathrm{A}}{=} i=1,2, \ldots, N$. Let $x_{i}$ be the corresponding eigenvector of $\lambda_{i}$. By definition, we have

$$
\underline{\mathrm{H}} \stackrel{\mathrm{A}}{=} x_{i}=\lambda_{i} x_{i} \text {. }
$$

Substituting $\stackrel{\mathrm{A}}{=}=\underline{\underline{\underline{L}}}^{T} \underline{\underline{\mathrm{L}} \text { into }}$ (4.3), we obtain

$$
\underline{\underline{\mathrm{H}}} \underline{\mathrm{L}}^{T} \underline{\underline{\mathrm{L}}} x_{i}=\lambda_{i} x_{\mathrm{i}}
$$

By definition, $x_{i}$ is a nonzero vector satisfying (4.3), hence the right-hand side of (4.4) cannot vanish. This implies that $\underline{\underline{L}} x_{i}$ is a nonzero vector (proof by contradiction). Therefore it makes sense to premultiply both sides of (4.4) by the singular matrix $\underline{\underline{L}}$ :

$$
\underline{\underline{\mathrm{L}}} \underline{\underline{\mathrm{H}}} \underline{\mathrm{L}}^{T} \underline{\underline{\mathrm{L}}} x_{i}=\lambda_{i} \stackrel{\mathrm{L}}{\underline{\underline{x}}} x_{i}
$$

Denoting $\underline{\underline{\mathrm{L}}} x_{i}$ by a nonzero vector $y_{i}$, we obtain

$$
\underline{\underline{\mathrm{L}}} \underline{\underline{\mathrm{H}}} \underline{\underline{L}}^{T} y_{i}=\lambda_{i} y_{i}
$$

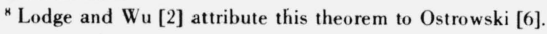


Equations (4.3) and (4.6) show that the nonzero eigenvalues of $\underline{\underline{H}} \underline{=}$ and $\underline{\underline{L}} \underline{\underline{H}} \stackrel{\mathrm{L}}{=}^{T}$ are identical. Some algebraic manipulation with $\underline{\underline{\mathrm{L}}} \underline{\underline{\mathrm{H}}} \underline{\underline{L}}^{T}$, which is symmetric, shows that its nonzero eigenvalues are given by those of $\underline{\underline{S}}$ with components $S_{i j}$ defined in (4.1).

\section{Significance of Results}

To discuss the significance of our mathematical results, it is appropriate here to introduce a closed-form solution of the distribution function $\psi$, originally due to Peterlin [1, p. 278 footnote], for a specific linear laminar flow with a constant transverse velocity gradient $\kappa$ expressible through a nondimensional gradient parameter $\beta:^{9}$

$$
\psi=\psi_{1} \psi_{2} \ldots \ldots \psi_{i} \ldots \ldots \psi_{N}
$$

where

$$
\psi_{i}=C_{i} \exp \left\{-\frac{3}{2} \frac{\mu_{\mathrm{i}}}{1+\beta^{2} / \lambda_{i}^{2}}\left[\xi_{i}^{2}-\frac{2 \beta \xi_{i} \zeta_{i}}{\lambda_{i}}+\left(1+\frac{2 \beta^{2}}{\lambda_{i}^{2}}\right) \zeta_{i}^{2}+\left(1+\frac{\beta^{2}}{\lambda_{i}^{2}}\right) \eta_{\mathrm{i}}^{2}\right]\right\},
$$

$C_{i}=\left(\mu_{i} / \pi\right)^{3 / 2}\left(1+\beta^{2} / \lambda_{i}^{2}\right)^{-1 / 2}$, and $\lambda_{\mathrm{i}}, \mu_{\mathrm{i}}, i=1,2, \ldots, N$, are the nonzero diagonal elements of matrices $\underline{\underline{\Lambda}}$ and $\underline{\underline{\mathrm{M}}}$ as defined in (1.3) and (1.4), respectively. The symbols $\xi_{i}, \zeta_{i}, \eta_{i}$ in (6.2) denote the nondimensionalized transformed coordinates of the $(i+1)$ th bead as defined below:

$$
\begin{aligned}
& \xi_{i}=\sum_{j=1}^{N} b_{0}^{-1}\left[Q^{-1}\right]_{i j} x_{j} . \\
& \zeta_{i}=\sum_{j=1}^{N} b_{0}^{-1}\left[Q^{-1}\right]_{i j} y_{j} . \\
& \eta_{\mathrm{i}}=\sum_{j=1}^{N} b_{0}^{-1}\left[Q^{-1}\right]_{i j} z_{\mathrm{j}} .
\end{aligned}
$$

The principal result of this paper has been the demonstration that the diagonal elements of both matrices $\underline{\underline{\Lambda}}$ and $\underline{\underline{\mathrm{M}}}$ are identical for each $i, i=0,1,2, \ldots, N$, if the hydrodynamic interaction parameter $h^{*}$ is less than 0.547 . This means that once we have computed the values of $\lambda_{i}, i=0,1,2$, ..., $N$, from the eigenvalue problem of the matrix product $\underline{\underline{\mathrm{H}}} \stackrel{\mathrm{A}}{=}$ as defined in (1.3), it is no longer necessary, as understood previously by Zimm [1], to compute (Q explicitly in order to calculate the diagonal elements $\mu_{i}, i=0,1,2, \ldots, N$, of the matrix $\underline{\underline{\mathrm{M}}}$ as defined in (1.4).

The result $\lambda_{i}=\mu_{i}, i=0,1,2, \ldots, N$, for $h^{*}<0.547$ is also significant in discrediting some computational results in the literature such as Zimm, Roe, and Epstein [7], etc., where the transformation matrix $\underline{Q}$, being not explicitly known, was expeditiously approximated by the orthogonal transformation matrix $\underline{\underline{Q}}_{R}$ of the well-known Rouse model of zero hydrodynamic interaction (i.e., $\underline{\underline{\mathrm{H}}}=\underline{\underline{1}})$. Under that circumstance, the matrix $\underline{\underline{\mathrm{M}}}$ is given by $\underline{\underline{Q}}{ }_{R}^{T} \underline{\underline{A}} \underline{\underline{Q}} R$ or

${ }^{9}$ The flow field is specified by the velocity vector $\mathbf{v}=(\kappa y, 0,0)$, and the parameter $\beta$ is related to $\kappa$ by the formula $\beta=\pi a_{h} b_{i}^{2} \kappa /(k T)$. whese $k$ is Boltzman's constant and $T$ is the absolute temperature.

${ }^{10}$ See, e.g., Zimm, Roe and Epstein [7]. Hearst [8], and Tschoegl [9]. 
$\underline{\underline{Q}}^{-1} \stackrel{\mathrm{A}}{=} \underline{\underline{Q}}_{R}$, and the eigenvalues $\mu_{j}$ are given by $4 \sin ^{2}[j \pi / 2(N+1)]$ which contradict our exact result with $\lambda_{j}=\mu_{j}$, and $\underline{\underline{M}}^{-1} \underline{\underline{\Lambda}}=\underline{\underline{N}}=\underline{\underline{1}}$ as shown in (3.4).

Computationally speaking, we are interested in the solution of the eigenvalue problem (4.3), for large $N$, and particularly, in the smaller values of $\lambda_{i}$. The equivalence statement of Lodge and $\mathrm{Wu}$ [2] provides the basis for computing those eigenvalues very economically with the aid of a large-scale computer. The exact computer-generated calculation renders many earlier approximation schemes obsolete. ${ }^{10}$ Without the benefit of the equivalence statement, Thurston and Morrison [10] used a computer to obtain the eigenvalues of the matrix product $\underline{\underline{\mathrm{H}}} \stackrel{\mathrm{A}}{=}$ for values of $N$ up to 15 . Based on their equivalence statement, Lodge and $\mathrm{Wu}$ [11] were able to extend the computational capability and reported some numerical results for bead/spring models with as many as 300 links. Unfortunately, Lodge and $\mathrm{Wu}$ did not exhibit all eigenvalues, nor the computer program with which eigenvalues could be generated for interesting applications. In the appendix we list a Fortran program and output to illustrate the significance of this paper and to enable others to extend these calculations, if they so desire.

We are indebted to Morris Newman, Senior Research Mathematician, National Bureau of Standards, Washington, D.C., for substantial original contribution and critical comments during the course of this investigation. We also wish to thank Martin Cordes of NBS Applied Mathematics Division for technical consultation related to the computational aspect of this work.

\section{References}

[ 1] Zimm, B. H., J Chem. Phys. 24, 269 (19.56).

[ 2] Lodge, A. S., and Wu, Y. -j., Rheol. Acta, 10, 539 (1971)

[ 3] Hohn, F. E., Elementary Matrix Algebra (MacMillan, 1958).

[ 4] Schwarz, H. R., Numerical Analysis of Symmetric Matrices, translated from the German by P. Hertelendy (Prentice-Hall, 1973).

[ 5] Halmos, P. R., Finite-Dimensional Vector Spaces (Van Nostrand, 1958).

[ 6] Ostrowski, A., Jber. Deutsch. Math. Verein. 60, 40 (19.57).

[ 7] Zimm, B. H., Roe, G. M., and Epstein, L. F., J. Chem. Phys. 24, 279, (1956).

[ 8] Hearst, J. E., J. Chem. Phys. 37, 2547 (1962).

[ 9] Tschoegl, N. W., J. Chem. Phys. 39, 149 (1963).

[10] Thurston, G. B., and Morrison, J. D., Polymer 10, 421 (1969).

[11] Lodge, A. S., and Wu, Y. -j., University of Wisconsin-Mathematics Research Center Report No. 1250. Madison, Wisconsin (1972).

\section{Appendix}

The following is a Fortran $\mathrm{V}$ driver program as executed on a UNIVAC-1108 Exec 8 computer at National Bureau of Standards Laboratory at Gaithersburg, Md. To simplify our programming effort, we take advantage of the well-documented subroutines furnished by the International Mathematical and Statistical Libraries, Inc., of Houston, Texas. For a complete description of these subroutines used in our program, the reader may consult the reference manual entitled "IMSL Library 2, Edition 4 (FORTRAN V), UNIVAC 1100 Series, Reference Manual, November 1974." The appearance of trade names such as UNIVAC and IMSL does not imply an endorsement of their product or services. 


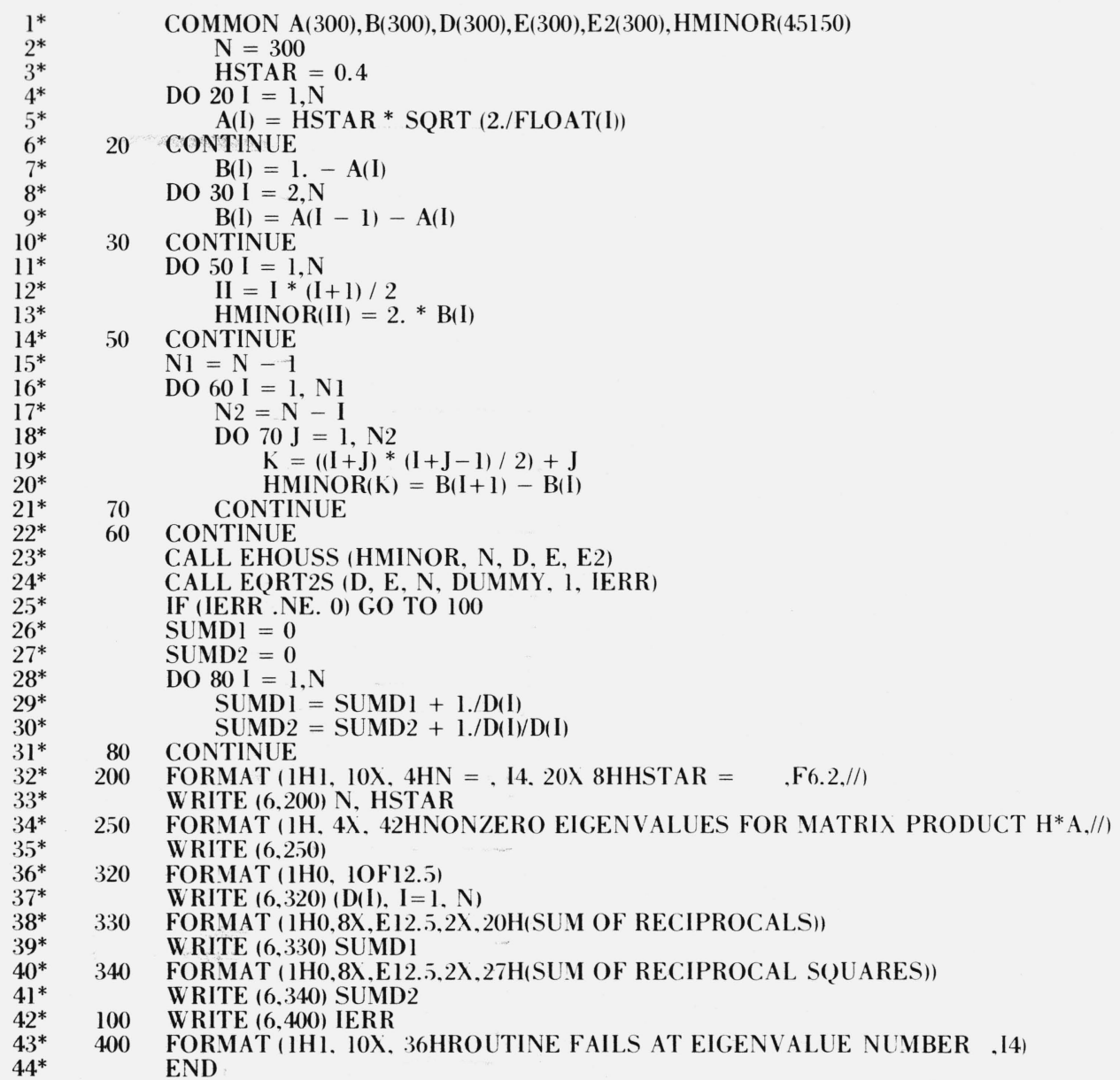


For $N=300$, and $h^{*}=0.4$ (corresponding to $a=0.566$ ), the following output was obtained at a total run cost of $\$ 47.91$ for a total run time of 5 min $42 \mathrm{~s}$. The CPU cost was $\$ 24.21$ with a run time of $5 \mathrm{~min} 36 \mathrm{~s}$.

\begin{tabular}{|c|c|c|c|c|c|c|c|c|c|}
\hline \multicolumn{10}{|c|}{$\begin{array}{l}\mathrm{N}=300 \quad \text { HSTAR }=.40 \\
\text { NONZERO EIGENVALUES FOR MATRIX PRODUCT H*A }\end{array}$} \\
\hline $11539-02$ & $.35945-02$ & $67180-02$ & 10383-01 & $.14480-01$ & $.18953-01$ & $.23749-01$ & $.28837-01$ & $.34185-01$ & $72-01$ \\
\hline $.45574-01$ & $.51576-01$ & & $.64119-01$ & $.70634-01$ & $.77297-01$ & $.84098-01$ & $.91028-01$ & $.98078-01$ & $.10524+00$ \\
\hline $.11251+00$ & $.11988+00$ & $.12734+00$ & $.13489+00$ & $.14252+00$ & $.15023+00$ & $.15801+00$ & $.16586+00$ & $.17377+00$ & $.18174+00$ \\
\hline $.18976+00$ & $.19784+00$ & $.20596+00$ & $.21412+00$ & $.22233+00$ & $.23058+00$ & $.23886+00$ & $.24717+00$ & $.25 .5 .51+00$ & $.26387+00$ \\
\hline $.27226+00$ & $.28067+00$ & $.28909+00$ & $.29754+00$ & $.30599+00$ & $.31446+00$ & $.32294+00$ & $.33142+00$ & $.33991+00$ & $.34840+00$ \\
\hline $.35690+00$ & $.36539+00$ & $.37388+00$ & $.38236+00$ & $.39084+00$ & $.39932+00$ & $.40778+00$ & $.41623+00$ & $.42467+00$ & $.43309+00$ \\
\hline & $.44990+00$ & & & $7496+00$ & & & $.49983+00$ & & $29+00$ \\
\hline $447+00$ & $.53263+00$ & $.54075+00$ & $.54885+00$ & $5691+00$ & $.56495+00$ & $.57294+00$ & $.58091+00$ & $.58883+00$ & $73+00$ \\
\hline & $61240+00$ & & $62791+00$ & $.63531+00$ & $64326+00$ & & $.65845+00$ & & $47+00$ \\
\hline & & & & & & & & & \\
\hline $.75277+00$ & $.75969+00$ & $.76656+00$ & $.77338+00$ & $.78015+00$ & $586+00$ & & $.80014+00$ & & $21+00$ \\
\hline & $.82608+00$ & & $.83872+00$ & $.84497+$ & $16+00$ & .85 & $.86338+00$ & & $8+00$ \\
\hline & & & & & & & & & \\
\hline $.93748+00$ & $.94279+00$ & $.94806+00$ & $.95326+00$ & $.95841+00$ & $51+00$ & & $53+00$ & & $4+00$ \\
\hline $.98816+00$ & $.99293+00$ & $.9976 .5+00$ & $.10023+01$ & $10069+$ & & .10 & +01 & .10 & $1+01$ \\
\hline & & & & & & & & & \\
\hline & $.10771+01$ & $.10808+01$ & $.10844+01$ & $.10880+$ & & .10 & $4+01$ & & $1+01$ \\
\hline & & .111 & $.11179+01$ & $.11210+$ & .11 & .11 & $99+01$ & & $7+01$ \\
\hline+01 & $3+01$ & $10+01$ & $7+01$ & & $9+01$ & & 01 & & \\
\hline & $66+01$ & $89+01$ & $.11712+01$ & $34+01$ & $756+01$ & .11 & $99+01$ & .118 & $0+01$ \\
\hline & $.11880+01$ & & & & & & & & \\
\hline .12 & $.12058+01$ & $.12073+01$ & $.12089+01$ & $.12104+01$ & $19+01$ & .121 & $8+01$ & .12 & $5+01$ \\
\hline & $.12203+01$ & $.12216+01$ & $.12229+01$ & $.12241+01$ & $.12253+01$ & $.1226 .5+01$ & $.12277+01$ & $.12288+$ & $9+01$ \\
\hline & $.12321+01$ & $.12331+01$ & $.12341+01$ & $.123 .51+($ & $.12361+($ & .123 & $.12379+01$ & $.12388+$ & \\
\hline $.12435+01$ & $.12414+01$ & $.12422+01$ & $.12430+01$ & $.12438+01$ & $.12445+01$ & $.12452+01$ & $.12460+01$ & $.12466+$ & $73+01$ \\
\hline & $.12486+01$ & $.12492+01$ & $.12492+01$ & $.12504+01$ & $.12510+01$ & $.12515+$ & $.12521+01$ & $.12526+$ & $31+01$ \\
\hline $.12536+01$ & $.12541+01$ & $.12545+01$ & $.125 .50+01$ & $.125 .54+01$ & $.125 .58+01$ & $.12562+01$ & $.12566+01$ & $.12570+$ & $73+01$ \\
\hline $.12577+01$ & $.12580+01$ & $.12583+01$ & $.12586+01$ & $.12589+01$ & $.12592+01$ & $.1259 .5+01$ & $.12597+01$ & $.12600+01$ & $.12602+01$ \\
\hline & $.12606+01$ & $.12608+01$ & $.12610+01$ & & $.12614+01$ & $.1261 .5+01$ & $.12617+01$ & $.12618+01$ & $.12619+01$ \\
\hline $.12670+01$ & $.12621+01$ & $.12622+01$ & $.12623+01$ & $.12674+01$ & $.12624+01$ & $.12625+01$ & $.12625+01$ & $.12625+01$ & $.12625+01$ \\
\hline
\end{tabular}

By changing the first three statements of the original program, one can find the eigenvalues for any $N$ and $h^{*}$ as long as the core storage is not exceeded and the value of $h^{*}$ is less than 0.547 . To illustrate this point, we show below for $N=250$ and $h^{*}=0.3$ the first three statements of the modified program and the appropriate output. The total cost for the run was $\$ 2.29$ with a total run time of 3 min $19 \mathrm{~s}$. The CPU cost was $\$ 13.96$ and the CPU time was 3 min $14 \mathrm{~s}$.

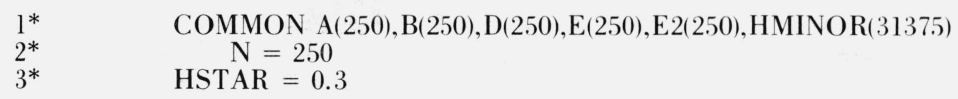


$\mathrm{N}=250 \quad$ HSTAR $=.30$

NONZERO EIGENVALUES FOR MATRIX PRODUCT H*A

\begin{tabular}{|c|c|c|c|c|c|c|c|c|c|}
\hline $.11737-02$ & $.36804-02$ & $.69245-02$ & $.10767-01$ & $.15099-01$ & $.19865-01$ & $.25015-01$ & $.30516-01$ & $.36336-01$ & $.424 .55-01$ \\
\hline $.48849-01$ & $.55504-01$ & $.62402-01$ & $.69531-01$ & $.76877-01$ & $.84432-01$ & $.92184-01$ & $.10012+00$ & $.10824+00$ & $.11653+00$ \\
\hline $.12499+00$ & $.13360+00$ & $.14237+00$ & $.15128+00$ & $.16033+00$ & $.16951+00$ & $.17882+00$ & $.18826+00$ & $.19781+00$ & $20748+00$ \\
\hline $.21726+00$ & $.22714+00$ & $.23713+00$ & $.24721+00$ & $.25739+00$ & $.26766+00$ & $.27802+00$ & $.28846+00$ & $.29898+00$ & $.30958+00$ \\
\hline $.32025+00$ & $.33099+00$ & $.34180+00$ & $.35268+00$ & $.36362+00$ & $.37462+00$ & $.38568+00$ & $.39679+00$ & $.40795+00$ & $.41916+00$ \\
\hline $.43042+00$ & $.44173+00$ & $.45308+00$ & $.46446+00$ & $.47589+00$ & $.48735+00$ & $.49894+00$ & $.51037+00$ & $.52192+00$ & $.53350+00$ \\
\hline $.54511+00$ & $.55674+00$ & $.56039+00$ & $.58006+00$ & $.59174+00$ & $.60345+00$ & $.61516+00$ & $.62689+00$ & $.63863+00$ & $.65038+00$ \\
\hline $.66213+00$ & $.67389+00$ & $.68565+00$ & $.69742+00$ & $.70918+00$ & $.72094+00$ & $.73270+00$ & $.74446+00$ & $.75621+00$ & $.76795+00$ \\
\hline $.77969+00$ & $.79141+00$ & $.80312+00$ & $.81482+00$ & $.82650+00$ & $.83817+00$ & $.84982+00$ & $.86145+00$ & $.87306+00$ & $.88465+00$ \\
\hline $.89622+00$ & $.90776+00$ & $.91928+00$ & $.93077+00$ & $.94224+00$ & $.95368+00$ & $.96508+00$ & $.97646+00$ & $.98780+00$ & $.99912+00$ \\
\hline $.10104+01$ & $.10216+01$ & $.10328+01$ & $.10440+01$ & $.10551+01$ & $.10662+01$ & $.10773+01$ & $.10883+01$ & $.10993+01$ & $.11102+01$ \\
\hline $.11211+01$ & $.11319+01$ & $.11427+01$ & $.11534+01$ & $.11641+01$ & $.11748+01$ & $.118 .54+01$ & $.119 .59+01$ & $.12064+01$ & $.12168+01$ \\
\hline $.12272+01$ & $.12376+01$ & $.12478+01$ & $.12581+01$ & $.12682+01$ & $.12783+01$ & $.12884+01$ & $.12984+01$ & $.13083+01$ & $.13182+01$ \\
\hline $.13280+01$ & $.13378+01$ & $.13475+01$ & $.13571+01$ & $.13667+01$ & $.13762+01$ & $.13856+01$ & $.13950+01$ & $.14043+01$ & $.14136+01$ \\
\hline $.14228+01$ & $.14319+01$ & $.14409+01$ & $.14499+01$ & $.14588+01$ & .146 & $.14764+01$ & $.14851+01$ & $.14938+01$ & $23+01$ \\
\hline $.15108+01$ & $.15192+01$ & $.15276+01$ & $.15358+01$ & $.15440+01$ & $.15 .521+01$ & $.15602+01$ & $.15682+01$ & $.15761+01$ & $.15839+01$ \\
\hline $.15916+01$ & $.15993+01$ & $.16069+01$ & $.16144+01$ & $.16218+01$ & $.16292+01$ & $.16365+01$ & $.16437+01$ & $.16508+01$ & $.16579+01$ \\
\hline $.16648+01$ & $.16717+01$ & $.16785+01$ & $.16853+01$ & & $.16905+$ & .17 & $14+01$ & .1717 & $39+01$ \\
\hline $.17301+01$ & $.17362+01$ & $.17421+01$ & $.17481+01$ & $.17539+01$ & $.17596+01$ & $.176 .53+01$ & $.17709+01$ & $.17764+01$ & $.17818+01$ \\
\hline $.17871+01$ & $.17923+01$ & $.17975+01$ & $.18025+01$ & $.18075+01$ & $.18124+01$ & $.18172+01$ & $.18220+01$ & $.18266+01$ & $.18312+01$ \\
\hline $.18356+01$ & $.18400+01$ & $.18443+01$ & $.18485+01$ & $.18526+01$ & $.18567+01$ & $.18606+01$ & $.18645+01$ & $.18683+01$ & $.18719+01$ \\
\hline $.1875 .5+01$ & $.18790+01$ & $.18825+01$ & $.18858+01$ & $.18891+01$ & $.18922+01$ & $.189 .53+01$ & $.18983+01$ & $.19012+01$ & $.19040+01$ \\
\hline $.19067+01$ & $.19093+01$ & $.19119+01$ & $.19143+01$ & $.19167+01$ & $.19190+01$ & $.19212+01$ & $.19233+01$ & $.19253+01$ & $.19272+01$ \\
\hline $.19290+01$ & $.19308+01$ & $.19324+01$ & $.19340+01$ & $.19355+01$ & $.19368+01$ & $.19381+01$ & $.19393+01$ & $.19405+01$ & $.19415+01$ \\
\hline $.19424+01$ & $.19433+01$ & $.19440+01$ & $.19447+01$ & $.19453+01$ & $.194 .58+01$ & $.19462+01$ & $.1946 .5+01$ & $.19467+01$ & $.19469+01$ \\
\hline
\end{tabular}

(Paper 80A2-443) 